\title{
Asociación del parasitismo por Profilicollis bullocki (Paleacanthocephala, Polymorphidae) con la conducta y la pigmentación de Emerita analoga (Anomura, Hippidae) en Chile
}

Association of parasitism by Profilicollis bullocki (Paleacanthocephala, Polymorphidae) with the behavior and pigmentation of Emerita analoga (Anomura, Hippidae) in Chile

\section{Rodolfo Jerez $^{1}$ y Mario George-Nascimento ${ }^{1}$}

\begin{abstract}
${ }^{1}$ Departamento de Ecología Costera, Facultad de Ciencias, Universidad Católica de la Santísima Concepción, Casilla 297, Concepción, Chile mgeorgen@ucsc.cl
\end{abstract}

\begin{abstract}
The intermediate host of the acanthocephalan Profilicollis bullocki in Chile is the mole crab Emerita analoga, a dominant species in sandy beaches, whereas the final hosts are marine birds. This parasite may affect the host phenotype because several studies about intermediate host-parasite systems where acanthocephalans participate, indicate modifications in host behavior. We experimentally assess if the parasite is able to modify the behavior and carapace pigmentation of naturally infected mole crabs. Results suggest that P. bullocki induce changes in the $E$. analoga phenotype: hosts with more P. bullocki are less active and lighter in carapace pigmentation than those without or with less parasites. This might affect its ability to escape predators.
\end{abstract}

Key words: Parasitism, mole crab, acanthocephalan, host phenotype

\section{INTRODUCCIÓN}

En diversos sistemas parásito-hospedador en los que participan acantocéfalos se ha documentado alteraciones conductuales y morfológicas en su hospedador intermediario, los que generalmente son artrópodos. Los registros más frecuentes consideran cambios de comportamiento y pigmentación en el caparazón (Moore 2002, Kennedy 2006, Lefevre et al. 2008). Estas modificaciones del fenotipo pueden incrementar la susceptibilidad de los hospedadores intermediarios parasitados a la depredación por sus hospedadores definitivos (aunque no exclusivamente por ellos, ver Medoc \& Beisel 2008), favoreciendo que se complete el ciclo de vida del parásito (Thomas et al. 2005, Poulin 2007). Al afectar las interacciones tróficas, los parásitos pueden modificar el flujo energético en las interacciones depredador-presa (Lafferty 1999, Thompson et al. 2005), teniendo consecuencias en los procesos de estructuración comunitaria (Poulin 2007).

Emerita analoga (Stimpson, 1857) (Anomura, Hippidae) es un crustáceo hermafrodita protándrico (Subramoniam \& Gunamalai 2003) común y abundante en el intermareal medio e inferior de playas arenosas. Su distribución geográfica se extiende desde Alaska, USA $\left(58^{\circ} \mathrm{N}\right)$ hasta Aysén, Chile $\left(55^{\circ} \mathrm{S}\right)$, aunque está ausente en zonas tropicales (Tam et al. 1996). La densidad y tamaño corporal de E. analoga aumentan hacia los niveles inferiores del intermareal, en la zona de rompiente (Contreras et al. 1999, 2000, Jaramillo et al. 2000a).

En Perú y Chile Emerita analoga es un hospedador intermediario del acantocéfalo Profilicollis bullocki Mateo, Córdova \& Guzmán, 1982 (= P. altmani, ver Balboa et al. 2009), un parásito que en estado adulto habita en el intestino de aves marinas (Riquelme et al. 2006). Se desconoce si P. bullocki es capaz de inducir cambios en el fenotipo de E. analoga. En consecuencia, se evaluó si la conducta y la pigmentación del caparazón de E. analoga, naturalmente parasitados, están o no asociadas a la abundancia de $P$. bullocki.

\section{Material y Métodos}

En la primavera del 2004 se recolectaron 98 ejemplares de Emerita analoga desde Lenga, una playa arenosa de la región del Biobío, Chile ( $\left.36^{\circ} 45^{\prime} \mathrm{S}, 73^{\circ} 10^{\prime} \mathrm{W}\right)$. Los crustáceos fueron trasladados al laboratorio donde permanecieron en un acuario de vidrio, provisto de agua de mar y arena, y aclimatados por 15 días para estabilizar su sistema nervioso y endocrino (Haye \& Ojeda 1998). Todos los ejemplares se mantuvieron a temperatura 
ambiente $\left(12,0+1^{\circ} \mathrm{C}\right)$, alimentados una vez por día con alimento para peces de acuario y sometidos a cambios de agua cada dos días.

Terminado el período de aclimatación, los ejemplares fueron trasladados al acuario experimental un día antes del inicio de los registros en la misma agua en que estaban durante el último lapso de su aclimatación. Luego, se registró por $10 \mathrm{~min}$ la conducta a cada uno de los 98 ejemplares por separado con una videocámara. Las observaciones se hicieron en un acuario de vidrio provisto de arena y con aireación constante y se cronometró el tiempo que emplearon en distintas actividades.

Se registró el tiempo (en segundos) que Emerita analoga ocupó para permanecer bajo el sustrato, nadar en la columna de agua, alimentarse, y desplazarse en contacto con el sustrato. Luego, se categorizó la pigmentación dorsal del caparazón en cuatro niveles: + claro, claro, oscuro y + oscuro, mediante inspección visual.

Posterior a la observación conductual, se midió la longitud total $(\mathrm{mm})$ de cada ejemplar con un vernier de $0,1 \mathrm{~mm}$ de sensibilidad, y su sexo fue determinado considerando la presencia de pleópodos y posición del poro genital (Contreras et al. 1999). Posteriormente, el hemocele de los ejemplares fue examinado bajo lupa estereoscópica para detectar y cuantificar la presencia de parásitos. Finalmente, se calculó la prevalencia (porcentaje de hospedadores parasitados), la abundancia (número de parásitos por hospedador examinado, ver Bush et al. 1997).

La significancia estadística de las diferencias en prevalencia fue evaluada mediante la prueba de $\chi^{2}$. Un análisis de la covarianza permitió evaluar el efecto de la longitud total y del sexo de Emerita analoga sobre la abundancia de Profilicollis bullocki (Zar 1984). Se evaluó con análisis de correlación si el tiempo ocupado por $E$. analoga en distintas actividades estaba asociado a la abundancia de parásitos. La significancia estadística de las variaciones de la pigmentación del caparazón según la abundancia de $P$. bullocki y el sexo de E. analoga, fue evaluada con un análisis de regresión logística multinomial (modelo logit) en el que la pigmentación + oscuro del caparazón fue usada como categoría control. En este tipo de análisis la variable de respuesta está en escala nominal u ordinal y posee más de 2 categorías (Agresti 1996).

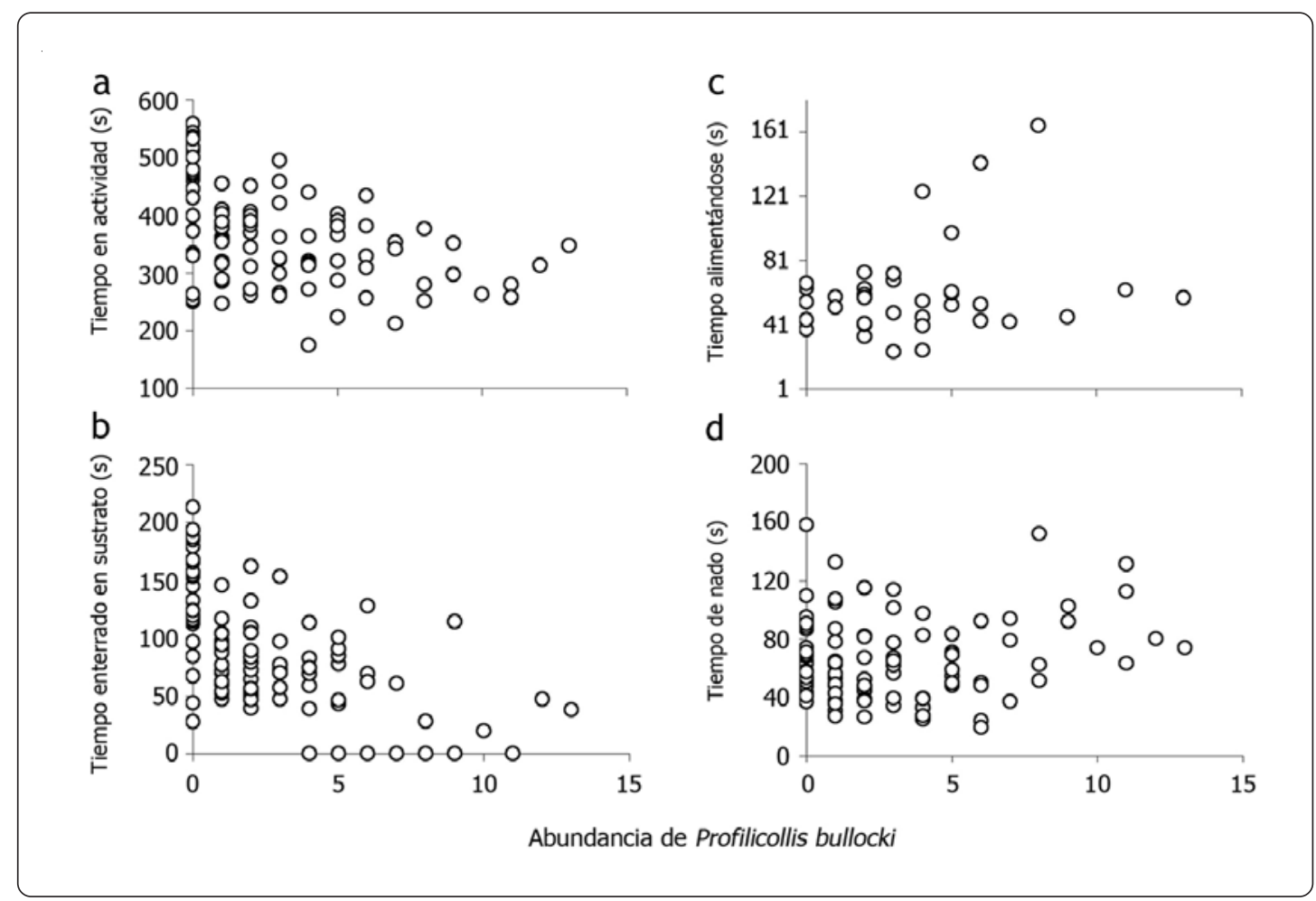

Figura 1. Diagramas de dispersión de la relación entre la abundancia de P. bullocki con el tiempo dedicado a distintas actividades efectuadas por Emerita analoga: a) actividad total, b) enterrado en la arena, c) alimentación, $\mathbf{y}$ d) nado / Dispersion diagrams of the relationship between the abundance of P. bullocki with time devoted by $E$. analoga to different activities: a) total activity, b) buried in the sand, c) feeding, and d) swimming 


\section{Resultados y Discusión}

El 75,5\% de los 98 ejemplares de Emerita analoga albergaban estados larvales de $P$. bullocki (abundancia 3,04 , d.e. $=3,20$, intensidad $=4,03$, d.e. $=3,10$, rango $=1$ 13). La longitud total de las hembras de E. analoga fue mayor que la de los machos $\left(\mathrm{F}_{(1,96)}=8,76, P<0,004\right)$. Asimismo, la prevalencia de Profilicollis bullocki fue mayor en las hembras $\left(\chi^{2}=5,02\right.$, g. $1 .=1, P=0,02$, Tabla 1). Sin embargo, el análisis de la covarianza de la abundancia de $P$. bullocki mostró que la pendiente de su relación con la longitud total del hospedador no era distinta entre sexos $\left(F_{1,94}=0,91, P=0,34\right)$, y que ni la longitud total $\left(\mathrm{F}_{1,94}=0,01, P=0,92\right)$, ni el sexo de $E$. analoga $\left(\mathrm{F}_{1,94}=0,72, P=0,39\right)$ eran factores relevantes para explicar su variabilidad.

El tiempo que Emerita analoga ocupó en estar activo disminuyó a medida que aumentaba la abundancia de parásitos ( $r=-0,521, P<0,001$, Fig. 1a), así como el tiempo ocupado en permanecer enterrado $(r=-0,225, P<$ $0,03$, Fig $1 \mathrm{~b})$, o desplazarse sobre el sustrato $(\mathrm{r}=-0,238$, $P<0,02)$. En cambio, el tiempo ocupado en alimentarse aumentó con la abundancia del acantocéfalo $(r=0,292, P$ $<0,003$, Fig 1c), y no se observó una correlación significativa entre la abundancia de $P$. bullocki y el tiempo que $E$. analoga ocupó en nadar $(\mathrm{r}=-0,054, P=0,597$, Fig. 1d).

Los resultados del análisis de regresión logística con la pigmentación del caparazón como variable de respuesta multinomial permiten afirmar que en general, la pigmentación del caparazón de Emerita analoga estaba asociada significativamente con la abundancia de $P$. bullocki $\left(\chi^{2}=14,64\right.$, g. l. $\left.=3, P=0,003\right)$, pero no con el sexo del crustáceo $\left(\chi^{2}=1,74\right.$, g.l. $\left.=3, P=0,628\right)$.
Tabla 1. Longitud total ( $\mathrm{mm})$ de E. analoga, prevalencia $(\mathrm{P}, \%)$, abundancia e intensidad promedio ( \pm d.e., desviación estándar del parasitismo por P. bullocki según el sexo de 98 individuos hospedadores / Total length $(\mathrm{mm})$ of $E$. analoga, prevalence $(P, \%)$, mean abundance and intensity ( \pm s.d., standard deviation) of $P$. bullocki according to the sex of 98 host individuals

\begin{tabular}{lcccc}
\hline & Longitud total & Prevalencia & Abundancia & Intensidad \\
\hline Machos, $\mathrm{n}=63$ & $14,01 \pm 1,77$ & 68,3 & $3,08 \pm 3,47$ & $4,51 \pm 3,34$ \\
Hembras, $\mathrm{n}=35$ & $15,03 \pm 1,34$ & 88,6 & $2,97 \pm 2,71$ & $3,35 \pm 2,64$ \\
\hline
\end{tabular}

Los resultados sugieren que Profilicollis bullocki modifica algunas expresiones fenotípicas de Emerita analoga, como son su actividad general que disminuye con la abundancia del acantocéfalo y la pigmentación del caparazón ya que a mayor abundancia de $P$. bullocki es más probable que el hospedador posea una pigmentación más clara. Por ejemplo, por cada cambio en una unidad en la abundancia de $P$. bullocki, el logaritmo de la razón entre las probabilidades de encontrar una pigmentación + claro y una pigmentación + oscuro, aumentó en 3,48 (Tabla 2).

La abundancia de Profilicollis bullocki podría incrementar la probabilidad de que E. analoga fuese consumida por un depredador, dado a que hospederos más parasitados presentaban menor actividad. Esto podría afectar su ritmo migratorio mareal (Jaramillo et al. 1993, 2000b), su capacidad de resistir el embate del oleaje, y su capacidad de emergencia explosiva dada por sus apéndices (Hernández et al. 2003), que en conjunto afectaría su habilidad de evitar a los depredadores.

La correlación positiva entre el tiempo que Emerita analoga ocupa en alimentarse y la abundancia de Profilicollis bullocki sugiere un aumento en sus requerimientos nutricionales, quizás para mantener

Tabla 2. Modelos de regresión logística multinomial para 4 categorías de pigmentación del caparazón de E. analoga según la abundancia de $P$. bullocki. Se indica el intercepto y la pendiente de los modelos junto a su error estándar, entre paréntesis, y al valor de Chi-cuadrado y de probabilidad para la hipótesis nula en que la pendiente es igual a cero / Models of multinomial logistic regression for 4 carapace pigmentation categories of E. analoga according to the abundance of P. bullocki. The intercept and the slope of the models are indicated with their standard error in parenthesis, together with the Chi-square value and the probability associated to a nule hypothesis of being not different from zero

\begin{tabular}{lccrr}
\hline Modelo & Intercepto & Pendiente & \multicolumn{1}{c}{$\chi^{2}$} & \multicolumn{1}{c}{$P$} \\
\hline $\log (\mathrm{P}+$ claro/P +oscuro $)$ & $-1,71(0,59)$ & $+3,48(1,02)$ & 11,59 & $<0,001$ \\
$\log (\mathrm{P}$ claro/P +oscuro $)$ & $-0,14(0,39)$ & $+1,70(0,84)$ & 4,08 & 0,045 \\
$\log (\mathrm{P}$ oscuro/P + oscuro $)$ & $-1,66(0,61)$ & $+2,52(1,10)$ & 5,23 & 0,022 \\
\hline
\end{tabular}


normales los niveles de glucosa y/u oxígeno en la hemolinfa (Crompton 1970), que eventualmente serían alterados por la presencia de cistacantos en la cavidad celómica, ya que los hospedadores intermediarios parasitados por acantocéfalos presentarían un aumento en el metabolismo (Haye \& Ojeda 1998) y en los niveles de dopamina y serotonina (Rojas \& Ojeda 2005).

Por otro lado, que la pigmentación del caparazón de Emerita analoga fuera más clara en los ejemplares que presentaron mayor abundancia de parásitos se contrapone a lo observado en otros sistemas acantocéfalo-hospedador invertebrado, ya que los hospedadores intermediarios parasitados tienden a presentar un color más intenso (Gotelli \& Moore 1992). Sin embargo, la despigmentación del caparazón de E. analoga también podría hacerlos más conspicuos y de más fácil detección por parte de los depredadores, en especial si se encuentra en sustratos contrastantes. En todo caso, sólo infecciones experimentales controladas podrían confirmar si las diferencias observadas son consecuencia de estar parasitados por P. bullocki (Poulin \& Thomas 1999, Thomas et al. 2005), ya que otros taxa parasitarios no considerados en este estudio podrían participar del fenómeno (Poulin et al. 2003).

\section{LITERATURA CITADA}

Agresti A. 1996. An introduction to categorical data analysis, 290 pp. John Wiley \& Sons, London.

Balboa L, A Hinojosa, C Riquelme, S Rodríguez, J Bustos \& M George-Nascimento. 2009. Alloxenic distribution of cystacanths of two Profilicollis species in sympatric crustacean hosts in Chile. Journal of Parasitology 95: 12051208.

Bush A, K Lafferty, J Lotz \& A Shostak. 1997. Parasitology meets ecology on its own terms: Margolis et al. revisited. Journal of Parasitology 83: 575-583.

Contreras H, O Defeo \& E Jaramillo. 1999. Life history of Emerita analoga (Stimpson) (Anomura, Hippidae) in a sandy beach of south central Chile. Estuarine, Coastal and Shelf Science 48: 101-112.

Contreras H, E Jaramillo \& P Quijón. 2000. Natural history of Emerita analoga (Stimpson) (Anomura, Hippidae) in a sandy beach of northern Chile. Revista Chilena de Historia Natural 73: 705-715.

Crompton DWT. 1970. An ecological approach to acanthocephalan physiology, 125 pp. Cambridge University Press, Cambridge.

Dezfuli B, B Maynard \& T Wellnitz. 2003. Activity levels and predator detection by amphipods infected with acanthocephalan parasite, Pomphorhynchus laevis. Folia Parasitologica 50: 129-134.
Gotelli N \& J Moore. 1992. Altered host behaviour in cockroach- acanthocephalan association. Animal Behaviour 43: 949-959.

Haye P \& FP Ojeda. 1998. Metabolic and behavioral alterations in the crab Hemigrapsus crenulatus (MilneEdwards 1837) induced by its acanthocephalan parasite Profilicollis antarcticus (Zdzitowiecki 1985). Journal of Experimental Marine Biology and Ecology 228: 73-82.

Hernández K, R González \& R Quiñones. 2003. Actividad de la láctico dehidrogenasa (LDH) en Emerita analoga (Stimpson, 1857) Crustacea, Hippidae. Gayana Zoología 67: 68-74.

Jaramillo E, C Duarte \& H Contreras. 2000a. Sandy beach macroinfauna from the coast of Ancud, Isla de Chiloé southern Chile. Revista Chilena de Historia Natural 73: 771-786.

Jaramillo E, J Dugan \& H Contreras. 2000b. Abundance, tidal movement, population structure and burrowing rate of Emerita analoga (Anomura, Hippidae) at dissipative and reflective sandy beach in south central Chile. Marine Ecology 21: 113-127.

Kennedy CR. 2006. Ecology of the Acanthocephala, 249 pp. Cambridge University Press, Cambridge.

Lafferty K. 1999. The evolution of trophic transmission. Parasitology Today 15: 111-115.

Lefevre T, C Labarbenchon, M Gauthier-Clerc, D Misse, R Poulin \& F Thomas. 2008. The ecological significance of manipulative parasites. Trends in Ecology and Evolution 24: 41-48.

Medoc V \& JN Beisel. 2008. An acanthocephalan parasite boosts the escape performance of its intermediate host facing non-host predators. Parasitology 135: 977-984.

Moore J. 2002. Parasites and the behavior of animals, 338 pp. Oxford University Press, Oxford.

Nickol BB, RW Heard \& N Smith. 2002. Acanthocephalans from crabs in the Southeastern U.S., with the first intermediate hosts known for Arhythmorhynchus frassoni and Hexaglandula corynosoma. Journal of Parasitology 88: 79-83.

Poulin R. 2007. Evolutionary ecology of parasites, 332 pp. Princeton University Press, Princeton.

Poulin R \& F Thomas. 1999. Phenotypic variability induced by parasites: extent and evolutionary implications. Parasitology Today 15: 28-32.

Poulin R, K Nichol \& A Latham. 2003. Host sharing and host manipulation by larval helminths in shore crabs: cooperation or conflict? International Journal for Parasitology 33: 425-433.

Riquelme C, M George-Nascimento \& L Balboa. 2006. Morfometría y fecundidad de Profilicollis bullocki Mateo, Córdova \& Guzmán 1982 (Acanthocephala: Polymorphidae) en especies simpátricas de aves costeras de Chile. Revista Chilena de Historia Natural 79: 465-474. 
Rojas J \& FP Ojeda. 2005. Altered dopamine levels induced by the parasite Profilicollis antarcticus on its intermediate host, the crab Hemigrapsus crenulatus. Biological Research 38: 259-266.

Subramoniam T \& V Gunamalai. 2003. Breeding biology of the intertidal sand crab Emerita (Decapoda: Anomura). Advances in Marine Biology 46: 91-182.

Tam YK, I Kornfield \& FP Ojeda. 1996. Divergence and zoogeography of mole crabs, Emerita spp. (Decapoda: Hippidae), in the Americas. Marine Biology 125: 489-497.
Thomas F, S Adamo \& J Moore. 2005. Parasitic manipulation: where are we and where should we go? Behavioural Processes 68: 185-199.

Thompson R, K Mouritsen \& R Poulin. 2005. Importance of parasites and their life cycle characteristics in determining the structure of a large marine food web. Journal of Animal Ecology 74: 77-85.

Zar JH. 1984. Biostatistical analysis, 718 pp. Prentice-Hall, Englewood Cliffs.

Recibido el 10 de diciembre de 2009 y aceptado el 7 de julio de 2010 